\title{
Crioconservación seminal de bagre rayado Pseudoplatystoma metaense (Teleostei, Pimelodidae), bajo diferentes protocolos de congelación ${ }^{\#}$
}

\author{
Seminal Cryopreservation of bagre rayado Pseudoplatystoma metaense \\ (Teleostei, Pimelodidae), under different protocols of freezing \\ JA Ramírez-Merlano*, VM Medina-Robles, PE Cruz-Casallas \\ Universidad de los Llanos, Grupo de Investigación sobre Reproducción y Toxicología de Organismos Acuáticos-GRITOX, \\ Instituto de Acuicultura, Universidad de los Llanos, Villavicencio, Meta, Colombia.
}

\begin{abstract}
SUMMARY
The aim was to determine the effect of different extenders and freezing rates on bagre rayado seminal quality. Sexually mature males $(1.5 \pm 0.2 \mathrm{~kg}$ and $56.6 \pm 2.9 \mathrm{~cm})$ and females $(2.8 \pm 0.7 \mathrm{~kg}$ and $71.3 \pm 6.1 \mathrm{~cm})$ were used. Ovulation and spermiation were induced with Carp Pituitary Extract. The semen was diluted (1:6, semen: diluent) with a glucose-based solution $(5.5 \%)$ and egg yolk (YH, $12 \%)$ or whole milk powder (LE, 5\%). As cryoprotectants dimethyl sulfoxide (DMSO, 10\%), methanol (MET, 12\%) or dimethyl acetamide (DMA, 10\%) were used. The diluted semen was packaged in $0.5 \mathrm{~mL}$ straws and frozen in two freezing curves $\left(\mathrm{C} 1=2.4{ }^{\circ} \mathrm{C} \cdot \mathrm{min}^{-1}\right.$ from 28 to $-4{ }^{\circ} \mathrm{C}$, followed by $10.0{ }^{\circ} \mathrm{C} \cdot \mathrm{min}^{-1}$ from -4 to $-80{ }^{\circ} \mathrm{C}$ and $\mathrm{C} 2=8.4{ }^{\circ} \mathrm{C} \cdot \mathrm{min}^{-1}$ of 4 to $-80^{\circ} \mathrm{C}$ ) and then immersed in liquid nitrogen $\left(-196^{\circ} \mathrm{C}\right)$. The straws were thawed in a water bath at $35^{\circ} \mathrm{C}$ by 60 sec and immediately assessed motility. The fertility was assessed through semination of 2 grams of oocytes with 100 or $400 \mu \mathrm{L}$ of fresh or frozen semen, respectively, and determined at 6:00 $\mathrm{h}$ postfertilization. The results showed higher rates of fertility with fresh semen, fertilized oocytes were observed in all treatments, with greater percentage (greater than $60 \%$ ) from the semen under the $\mathrm{C} 2$ (greater than $60 \%$ ) and 10 or $12 \%$ of DMSO or MET, respectively and $5.5 \%$ glucose and $5 \%$ of LE. These results allow to establish an acceptable freezing protocol for the artificial reproduction of bagre rayado.
\end{abstract}

Palabras clave: crioconservación, espermatozoide, Pseudoplatystoma metaense, semen

Key words: cryopreservation, spermatozoa, Pseudoplatystoma metaense, sperm.

\section{INTRODUCCIÓN}

La crioconservación ha sido reconocida como un método que contribuye a la reproducción asistida de varias especies de animales (Fabbrocini y col 2000) y como una herramienta esencial en los programas de mejoramiento animal, generando cambios significativos en esta industria animal (Drokin y col 1998). Sin embargo, las investigaciones hasta ahora realizadas han sido orientadas principalmente a evaluar diversas mezclas de sustancias crioprotectoras y tasas de congelación/descongelación del material seminal (Medina-Robles y col 2005). En peces, una amplia gama de sustancias crioprotectoras permeables a la membrana plasmática han sido estudiadas, siendo las más comunes el dimetil sulfóxido, glicerol y propilen glicol (Farrant 1970). Por otra parte, sustancias no permeables como azúcares (glucosa, fructosa, sacarosa) y proteínas (yema de huevo, leche en polvo, glicoproteínas) son frecuentemente añadidas a los diluyentes para estabilizar la actividad de la

Aceptado: 23.12.2010.

\# Proyecto de investigación apoyado por el Ministerio de Agricultura y Desarrollo Rural y el Instituto de Investigaciones de la Orinoquia Colombiana-IIOC. Contrato 015-1/06 MADR-CIAT-Universidad de los Llanos.

* Km 12 vía Puerto López, A.A. 110, Villavicencio, Meta, Colombia; juanantonioramirez.merlano@gmail.com membrana plasmática de los espermatozoides (De Leeuw y col 1993). Durante la crioconservación, la alta ración molar de colesterol o fosfolípidos y la baja proporción de ácidos grasos poliinsaturados le confieren al espermatozoide sensibilidad a la congelación (Darin-Bennet y White 1977), permitiendo que sólo un pequeño porcentaje permanezca viable después de dicho proceso (Ogier de Baulny y col 1997). Lo anterior ha sido atribuido a la baja pérdida de agua de las células, ocasionando la formación de cristales de hielo intracelular durante la congelación o descongelación (Viveiros 2005).

Para evaluar la viabilidad del semen después de la descongelación, el criterio más usado es la movilidad espermática. Sin embargo, en ensayos realizados con semen del bagre africano (Clarias gariepinus) se observó baja movilidad de las células espermáticas postdescongelación, pero una alta tasa de eclosión (Viveiros y col 2000), mostrando una relación no directa entre estas dos variables.

En Colombia se ha generado un creciente interés por las especies de bagres debido a su alto potencial para la piscicultura; sin embargo, son pocos los estudios realizados con estas especies, lo cual no ha permitido su cultivo a escala comercial, debido principalmente a las dificultades para lograr su reproducción en cautiverio y a su marcada estacionalidad reproductiva (Guerrero 2003). El bagre rayado (Pseudoplatystoma metaense) es una especie nativa colombiana considerada en peligro de extinción 
y potencialmente cultivable por su alto valor comercial (Mojica y col 2002).

Varios métodos han sido reportados para la crioconservación seminal de bagres, incluyendo al bagre rayado (Pinzón-Arciniegas y col 2005, Medina-Robles y col 2007, Ramírez-Merlano y col 2008); Ramdia sebae cf (Villalobos y Osorio 2007); Clarias gariepinus (Viveiros y col 2000) e Ictalurus furcatus (Paul y col 2003), con una alta variabilidad en los resultados observados. Por lo anterior, el objetivo del presente trabajo fue evaluar diferentes tasas de congelación y composición de los diluyentes sobre la calidad postdescongelación de espermatozoides de bagre rayado (Pseudoplatystoma metaense), con el fin de optimizar su reproducción en cautiverio.

\section{MATERIALES Y MÉTODOS}

\section{MATERIAL BIOLÓGICO}

Se utilizaron hembras $(2,8 \pm 0,7 \mathrm{~kg}$ y $71,3 \pm 6,1 \mathrm{~cm})$ y machos $(1,5 \pm 0,2 \mathrm{~kg}$ y $56,6 \pm 2,9 \mathrm{~cm}$ de peso corporal y longitud total, respectivamente) adultos de bagre rayado (Pseudoplatystoma metaense) nacidos en cautiverio, provenientes del plantel de reproductores de la estación piscícola de la Universidad de los Llanos y de otras estaciones piscícolas del departamento del Meta-Colombia. Los ejemplares fueron seleccionados teniendo en cuenta su condición de madurez sexual. Para el caso de las hembras, la madurez sexual se determinó mediante el examen de una biopsia para determinar el tamaño de los ovocitos y la posición de su vesícula germinal; en el caso de los machos, por la presencia de semen en la papila urogenital después de leve masaje cráneo-caudal, aplicado sobre las paredes de la cavidad celómica.

\section{INDUCCIÓN A LA MADURACIÓN FINAL DE LAS GÓNADAS}

A los machos se les administró una única inyección intramuscular de extracto de hipófisis de carpa (Stoller Fisheries, USA), equivalente a $4 \mathrm{mg} \cdot \mathrm{kg}^{-1}$ de peso corporal, aplicada en la parte caudal de la base de la aleta dorsal. Por su parte, las hembras recibieron una dosis total de $5,65 \mathrm{mg} \cdot \mathrm{kg}^{-1}$ de peso corporal, en tres inyecciones: 0,25 ; 0,4 y $5 \mathrm{mg} \cdot \mathrm{kg}^{-1}$ a las 0,24 y $36: 00 \mathrm{~h}$, respectivamente.

\section{OBTENCIÓN DEL SEMEN}

La extracción del semen se realizó 18:00 h después de la inyección hormonal. Los machos fueron previamente tranquilizados por inmersión durante 5 min en solución de 2-fenoxietanol (300 ppm, Sigma Chemical Co., St. Louis, Missouri, USA). Inmediatamente después de la pérdida del eje de nado, fueron retirados de la solución anestésica, secados cuidadosamente en la región genital, aplicando una leve presión para provocar la expulsión de orina y un masaje cráneo-caudal de la cavidad celómica para ocasionar la expulsión del semen. Este último fue colectado en tubos de vidrio aforados estériles para determinar directamente el volumen, evitando la contaminación con bilis, orina o heces; posteriormente fue mantenido a temperatura ambiente $\left(26 \pm 1^{\circ} \mathrm{C}\right)$.

\section{EVALUACIÓN DE LA CALIDAD SEMINAL}

La movilidad masal se evaluó de forma subjetiva, activando una alícuota de semen $(20 \mu \mathrm{L})$ con $180 \mu \mathrm{L}$ de agua destilada, lo cual fue realizado sobre una lámina excavada (1,0-1,2 mm de profundidad, Micro-Slides Premiere, China) montada en un microscopio óptico (10X de magnificación, Zeiss, Alemania). Inmediatamente después de la adición del agua destilada se evaluó la duración (seg) de la movilidad espermática (TA) hasta la inmovilidad del $90 \%$ de los espermatozoides. La concentración espermática fue determinada por recuento en cámara de Neubauer (40X), previa dilución del semen 1:4.000 con solución salina formolada $(0,9 \%$ de $\mathrm{NaCl}$ y formol al $3 \%)$. Para cada muestra, la evaluación se realizó por duplicado y el promedio fue utilizado para los análisis subsecuentes.

CRIOCONSERVACIÓN ESPERMÁTICA Y CONDICIONES DE CONGELACIÓN

Efecto del diluyente y la tasa de congelación. Se utilizaron únicamente muestras con movilidad superior a $80 \%$. Inicialmente las muestras seminales fueron diluidas en proporción 1:6 (semen: diluyente), manteniendo para todos los casos una proporción constante de glucosa (5,5\% p:v). Fueron evaluados dos crioprotectores no permeables: $12 \%$ de yema de huevo (YH v:v) y $5 \%$ de leche entera en polvo (LE p:v) y tres crioprotectores permeables: 10\% dimetilsulfóxido (DMSO v:v, Sigma Chemical Co., St. Louis, MO, USA), $12 \%$ metanol (MET v:v, Sigma Chemical Co., St. Louis, MO, USA) y 10\% dimetil acetamida (DMA v:v Merck Schuchardt OHG., Hohenbrunn, Germany). Se conformó un diseño factorial $2 \times 3 \times 2$ [dos crioprotectores externos (YH y LE), tres crioprotectores internos (DMSO, MET y DMA) y dos tasas de congelación (C1 y C2)], evaluando de esta manera 12 tratamientos. Inmediatamente después la mezcla semen: diluyente se empacó en pajillas de 0,5 mL (130x3 mm, Instruments de Médecine Vétérinaire, Minneapolis, USA) y se sometieron a dos curvas de congelación: la primera (C1) de 28 a $-4{ }^{\circ} \mathrm{C}$, con una tasa de descenso de $2,4{ }^{\circ} \mathrm{C} \cdot \mathrm{min}^{-1}$ seguido por $10,0^{\circ} \mathrm{C} \cdot \mathrm{min}^{-1} \mathrm{de}-4 \mathrm{a}-80{ }^{\circ} \mathrm{C}$ y una segunda $(\mathrm{C} 2)$ curva de congelación de 4 a $-80{ }^{\circ} \mathrm{C}$, equivalente a $8,4^{\circ} \mathrm{C} \cdot \mathrm{min}^{-1}$. Para las dos curvas, el tiempo de congelación fue de 20 y $15 \mathrm{~min}$, respectivamente. Lo anterior fue realizado en vapores de NL, utilizando un congelador programable (Ice Cube 15X, Sy-LAB Instruments GmbH, Austria). Posteriormente, todas las pajillas fueron removidas de la cámara de congelación y transferidas a un termo de almacenamiento (Taylor-Wharton HC 35, Theodoro, AL, 
USA), hasta la realización de la evaluación de la calidad seminal postdescongelación y de la fertilidad.

Evaluación de la movilidad y velocidad individual postdescongelación a través de un sistema de análisis espermático asistido por computador (CASA-Computer Assisted Sperm Analysis). Quince días postcongelación, el semen fue descongelado por inmersión durante $60 \mathrm{~s}$ en baño de agua a $35^{\circ} \mathrm{C}$; tanto la movilidad como la velocidad espermática individual fueron determinadas a través de un sistema de análisis espermático asistido por computador CASA (Medealab CASA-versión 5.4, Medea AVG, Alemania) empleando cámara de Makler (área de $1 \mathrm{~mm}^{2}$, SefiMedical Instruments, Israel) y microscopio óptico de contraste de fase (Nikon E400, Japón). El procedimiento consistió en colocar una alícuota de semen (c.a. $1 \mu \mathrm{L})$ en la cámara de Makler para determinar la movilidad espermática individual después de la activación con bicarbonato de sodio al $1 \%\left(\mathrm{NaHCO}_{3}\right)($ c.a. $20 \mu \mathrm{L})$ y la observación de aproximadamente 200 espermatozoides por campo. Las características seminales determinadas fueron: Movilidad progresiva lineal rápida (MPLR), Movilidad progresiva lineal lenta (MPLL), Movilidad local o nado en círculo (MLC), Inmóviles (IMV), Movilidad total espermática individual (MTI), Movilidad circular espermática (MC), Velocidad curvilínea (VCL), Velocidad en línea recta (VLR) y Velocidad promedio de desplazamiento (VAP). Las movilidades fueron expresadas en porcentaje (\%) y las velocidades en $\mu \mathrm{m} \cdot \mathrm{seg}^{-1}$.

Evaluación de las alteraciones morfológicas postdescongelación. Para evaluar alteraciones morfológicas postdescongelación el semen fue diluido en solución de formaldehído $35 \%$ (4\%)-citrato de sodio [2,9\%, $\mathrm{Na}_{2} \mathrm{H}$ $\left(\mathrm{C}_{3} \mathrm{H}_{5} \mathrm{O}(\mathrm{COO})_{3}\right]$ y agua destilada. El análisis morfológico fue realizado sobre 100 espermatozoides por tratamiento, bajo microscopio óptico (100x) de contraste de fase (Nikon E400, Japón), determinando alteraciones de cabeza (aislada, microcefalia y macrocefalia), pieza media (degenerada) y flagelo (fracturado y aislado).

Ensayos de fertilidad. De cada tratamiento fueron tomadas al azar pajillas que fueron descongeladas por inmersión en baño de agua a $35^{\circ} \mathrm{C}$ durante $60 \mathrm{~s}$, para luego evaluar la movilidad postdescongelación y realizar las pruebas de fertilidad. Fueron utilizados $2 \mathrm{~g}$ de oocitos, los cuales se seminaron con $400 \mu \mathrm{L}$ (c.a) de semen descongelado. Como control se utilizó la misma cantidad de ovocitos seminados con $100 \mu \mathrm{L}$ de semen fresco. Tanto el semen descongelado como fresco fue activado con $5 \mathrm{~mL}$ de solución de bicarbonato de sodio al $1 \%$. Después de 1 minuto, los huevos fueron lavados varias veces para remover el exceso de semen. Los huevos seminados fueron colocados en incubadoras experimentales con flujo continuo de agua de $2 \mathrm{~L}$ de capacidad. La fertilidad fue evaluada $6 \mathrm{~h}$ postseminación, calculando la proporción de oocitos fecundados (aspecto traslúcido y estado de cierre del blastoporo) en cada uno de los protocolos estudiados. El número de huevos por gramo, así como la concentración espermática en cada pajilla o tratamiento fue estimado para poder determinar la relación espermatozoides/ovocito utilizado.

Durante la incubación, se monitorearon los parámetros físico-químicos del agua por medio de una sonda multiparamétrica YSI (MPS YSI 556, Spanish. qxp), registrándose temperatura de $25,9 \pm 0,9{ }^{\circ} \mathrm{C}$, conductividad de $41 \pm 15,5$ $\mu \mathrm{s} \cdot \mathrm{cm}^{1}$ y $\mathrm{pH}$ de $6,2 \pm 0,4$.

\section{ANÁLISIS ESTADÍSTICO}

Todos los valores fueron expresados como media \pm error estándar de la media (EE). Para evaluar los efectos de cada tratamiento sobre las variables de movilidad y fertilidad, se empleó un análisis de varianza (ANOVA), seguido de la prueba de Tukey. Previo a cualquier análisis estadístico, los datos fueron sometidos a pruebas de Bartlet para determinar su homogeneidad y orientar el tipo de análisis estadístico a realizar. En todos los casos $\mathrm{P}<0,05$ fue utilizado como criterio estadístico para revelar diferencias significativas. Los datos fueron analizados con el software SAS versión 8,02 para Windows (1.999-2.001 por SAS Institute Inc, Cary, NC. USA).

\section{RESULTADOS}

El semen fresco se caracterizó por volumen de $10,14 \pm 1,83 \mathrm{~mL}$, movilidad mayor al $90 \%$, tiempo de activación de 48,9 $\pm 1,4 \mathrm{~s}$ y concentración de 35,18 $\pm 3,8$ $\mathrm{x} 10^{6}$ espermatozoides $/ \mu \mathrm{L}$.

\section{EFECTO DEL DILUYENTE Y DE LA TASA DE CONGELACIÓN}

El seguimiento de la curva de congelación C1 mostró un descenso lento de la temperatura desde $28^{\circ} \mathrm{C}$ hasta $-77{ }^{\circ} \mathrm{C}$ durante un periodo de $20 \mathrm{~min}$, en contraste a lo observado para la $\mathrm{C} 2$, donde esta misma temperatura fue alcanzada a los $11 \mathrm{~min}$. Para ambas curvas se presentó un fenómeno de ascenso de temperatura entre los -5 y $-15{ }^{\circ} \mathrm{C}$, estabilizándose la temperatura a los 20 min para la $\mathrm{C} 1$ y a los 15 min para la $\mathrm{C} 2$ (figura 1 ).

La figura 2 muestra la variación de la movilidad espermática durante todo el proceso de crioconservación, obsérvese en las figuras 2 a y 2 c la evaluación de la movilidad en diferentes fases, tomando como punto de inicio la movilidad del semen fresco (SF $95 \pm 0,0 \%$ ). El semen diluido con DMSO-YH y DMSO-LE mantuvo una movilidad cercana al SF. Sin embargo, en la fase 3 la movilidad descendió considerablemente a valores inferiores a $30 \%$, utilizando la curva programada C1 (figura 2a). Para las dos curvas evaluadas la movilidad disminuyó gradualmente durante el proceso de crioconservación.

El porcentaje de movilidad masal postdescongelación varió entre 0 y 23\%, para el semen sometido a 


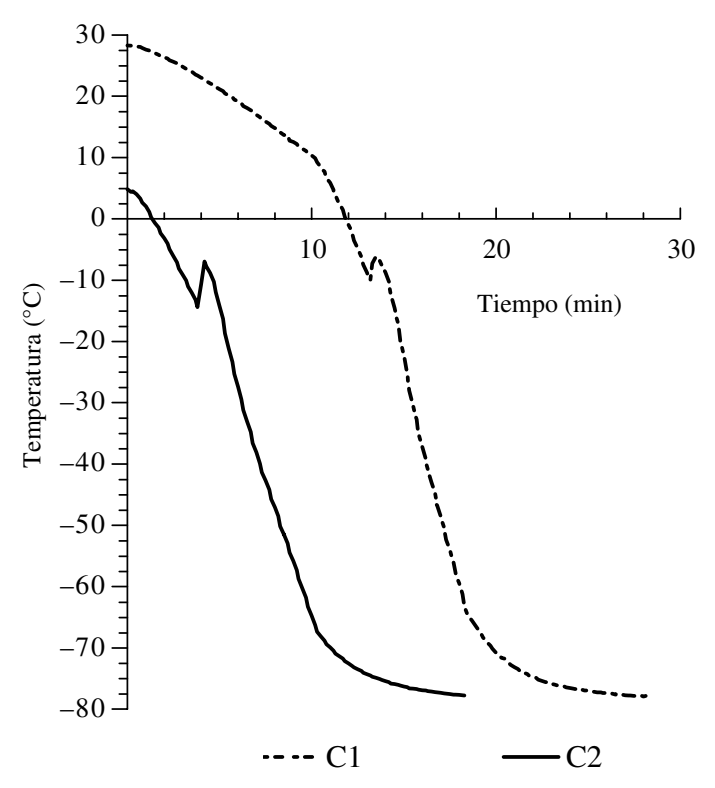

Figura 1. Variación de la temperatura de semen de bagre rayado (Pseudoplatystoma metaense), bajo diferentes protocolos de congelación en vapores de nitrógeno líquido usando un congelador programable. $\mathrm{C} 1=2{ }^{\circ} \mathrm{C} \cdot \mathrm{min}^{-1}$ de 28 a $-4{ }^{\circ} \mathrm{C}$, seguido por $8,2^{\circ} \mathrm{C} \cdot \mathrm{min}^{-1} \mathrm{de}-4 \mathrm{a}-80{ }^{\circ} \mathrm{C}$ (periodo de congelación 20 $\min$ ); $\mathrm{C} 2=8,2{ }^{\circ} \mathrm{C} \cdot \mathrm{min}^{-1}$ de $4 \mathrm{a}-80{ }^{\circ} \mathrm{C}$ (periodo de congelación $15 \mathrm{~min})$.

Temperature variation of bagre rayado (Pseudoplatystoma metaense) semen under different protocols of freezing in liquid nitrogen vapor using a programmable freezer. $\mathrm{C} 1=2.4^{\circ} \mathrm{C} \cdot \mathrm{min}^{-1}$ de $28 \mathrm{a}-4^{\circ} \mathrm{C}$, followed by $10.0^{\circ} \mathrm{C} \cdot \mathrm{min}^{-1} \mathrm{de}-4 \mathrm{a}-80^{\circ} \mathrm{C}$ (freezing period $20 \mathrm{~min}$ ); $\mathrm{C} 2=8.4{ }^{\circ} \mathrm{C} \cdot \mathrm{min}^{-1} \mathrm{de} 4 \mathrm{a}-80^{\circ} \mathrm{C}$ (freezing period $15 \mathrm{~min}$.).

congelación con la $\mathrm{C} 1$ (figura 2b), siendo la movilidad mayor para MET-YH $(23,3 \pm 5,4 \%)$, seguido de DMSO-LE $(22,5 \pm 4,4 \%)$, pero sin observarse diferencias significativas $(\mathrm{P}>0,05)$ entre estos tratamientos. En los tratamientos MET-LE, DMA-YH y DMA-LE, la movilidad fue menor en comparación con los diluyentes anteriormente mencionados con diferencias estadísticas $(\mathrm{P}<0,05)$ al ser comparados con estos. El semen congelado con la C2 mostró valores de movilidad masal superiores con relación a los observados en el semen congelado con la $\mathrm{C} 1$ (figura 2d). En la C2 el DMSO-LE mostró el porcentaje más alto de movilidad masal $(43,3 \pm 4,2 \%)$ siendo significativamente diferente $(\mathrm{P}<0,05)$ cuando se comparó con MET-YH, MET-LE, DMA-YH y DMA-LE.

\section{MOVILIDAD Y VELOCIDAD INDIVIDUAL}

POSTDESCONGELACIÓN A TRAVÉS DE UN SISTEMA

DE ANÁLISIS ASISTIDO POR COMPUTADOR

La MPLR del semen crioconservado presentó diferencias significativas con respecto al semen fresco $(\mathrm{P}<0,05)$, siendo mayor para este último $(52,7 \pm 4,6 \%)$. La MPLR para el semen crioconservado fue mayor con DMSO-LE sometido a la $\mathrm{C} 2(11,9 \pm 6,7 \%)$ sin diferencias significativas
$(\mathrm{P}<0,05)$ cuando fue comparada con los otros tratamientos (figura 3a). En contraste con lo mostrado en la MPLR, la MPLL (figura $3 b$ ) para el semen crioconservado fue menor al semen fresco $(34,7 \pm 2,2 \%)$, sin mostrar diferencias significativas $(\mathrm{P}>0,05)$ al ser comparadas con DMSOYH-C1, DMSO-YH-C2, DMSO-LE-C2, MET-YH-C1 y MET-YH-C2. La mayor MPLL fue observada para DMSOYH-C1 $(25,2 \pm 5,2 \%)$ sin diferencia significativa con los demás tratamientos $(\mathrm{P}>0,05)$. En general, los espermatozoides crioconservados presentaron movilidades en círculo mayores que el SF (figura 3c). El tratamiento MET-YH-C2, mostró un MLC significativamente mayor $(\mathrm{P}<0,05)$ con respecto a los demás tratamientos $(58,9 \pm 6,6 \%)$. Obsérvese que los tratamientos MET-LE-C1 (0\%), DMA-YH-C1 $(10,0 \pm 4,2 \%)$, DMA-LE-C1 $(21,6 \pm 5,0 \%)$ presentaron las MLC más bajas $(\mathrm{P}>0,05)$.

En cuanto al porcentaje de IMV (figura 3d) se destacan los tratamientos DMA-YH-C1 y DMA-LE-C1 con alta presencia de espermatozoides inmóviles $(84,5 \pm 3,6$ y $78,3 \pm 5,0 \%$, respectivamente) mostrando diferencias significativas al compararse con el SF $(4,3 \pm 1,6 \%)$ $(\mathrm{P}<0,05)$. La figura 3f muestra que la MC sólo fue observada en el tratamiento MET-LE-C2 $(0,1 \pm 0,1 \%)$, siendo significativamente diferente al control $(\mathrm{P}<0,05)$. La MTI postdescongelación para todos los tratamientos fue significativamente menor con respecto al SF (figura 3e), el cual presentó una movilidad mayor de $90 \%$. Por su parte el tratamiento DMSO-YH-C1 presentó la mayor MTI $(36,8 \pm 4,3 \%)$. Obsérvese en la figura 3 que los tratamientos MET-LE-C1, DMA-LE-C1 y DMA-LE-C2 no mostraron este tipo de movilidad individual.

\section{VELOCIDAD ESPERMÁTICA}

En todos los casos las velocidades determinadas con el CASA fueron significativamente menores $(\mathrm{P}<0,05)$ para el semen crioconservado. En cuanto a la VLR (figura 4a) esta fue significativamente mayor $(\mathrm{P}<0,05)$ en semen crioconservado con los tratamientos DMSO-LE-C2 y DMSO-YH-C1 $\left(8,4 \pm 3,2\right.$ y 7,4 $\pm 2,0 \mu \mathrm{m} . \mathrm{sg}^{-1}$, respectivamente). De igual forma, para estos mismos tratamientos se observaron los más altos valores para la VCL $(10,8 \pm 3,2$ y $10,1 \pm 7,3 \mu \mathrm{m} \cdot \mathrm{sg}^{-1}$, respectivamente) y VAP $(8,72 \pm 3,1 \mathrm{y}$ $7,9 \pm 1,9 \mu \mathrm{m} \cdot \mathrm{sg}^{-1}$, respectivamente), con un comportamiento contrario para el semen crioconservado con las sustancias MET y DMA en las tres variables de velocidad evaluadas (figuras $4 \mathrm{~b}$ y $4 \mathrm{c}$ ).

\section{ALTERACIONES MORFOLÓGICAS POSTDESCONGELACIÓN}

La figura 5 muestra algunas alteraciones morfológicas observadas en semen de bagre rayado postdescongelación. En todos los tratamientos fue notoria la presencia de cabezas y flagelos aislados (b y c, respectivamente). La presencia de macrocefalia $(\mathrm{k}, \mathrm{l})$ solo fue observada en semen crioconservado con los tratamientos DMSO- 
a

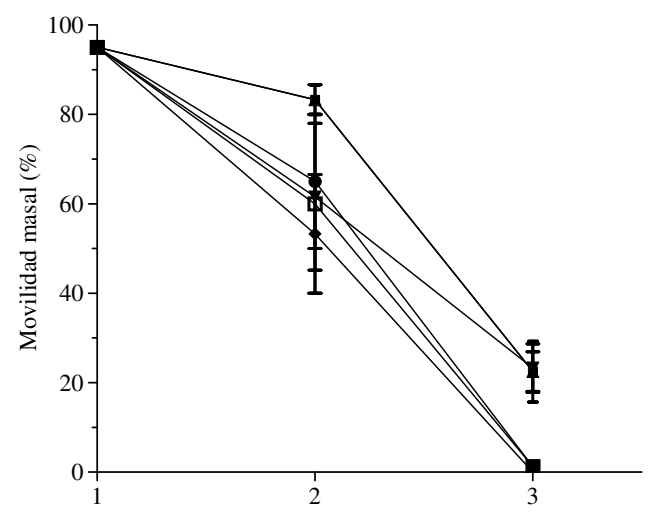

c

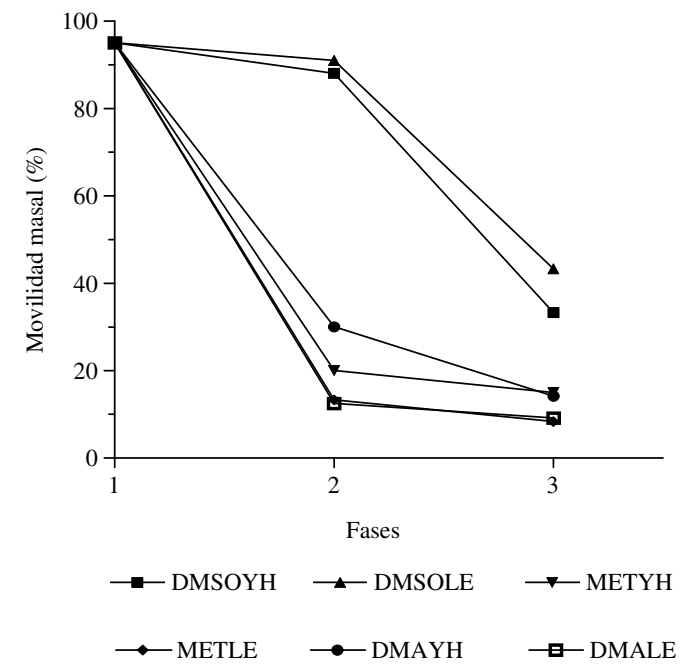

$\mathrm{b}$

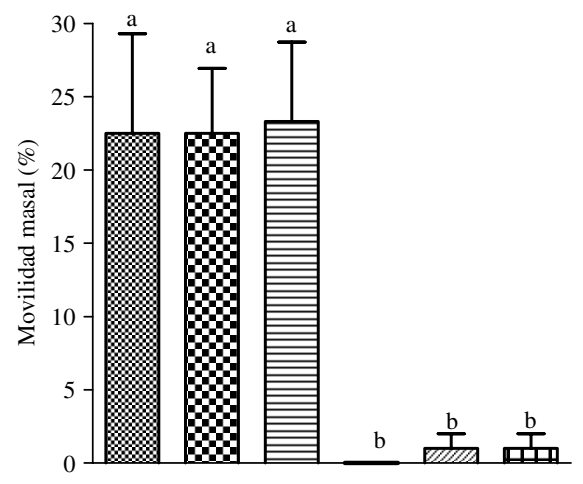

d

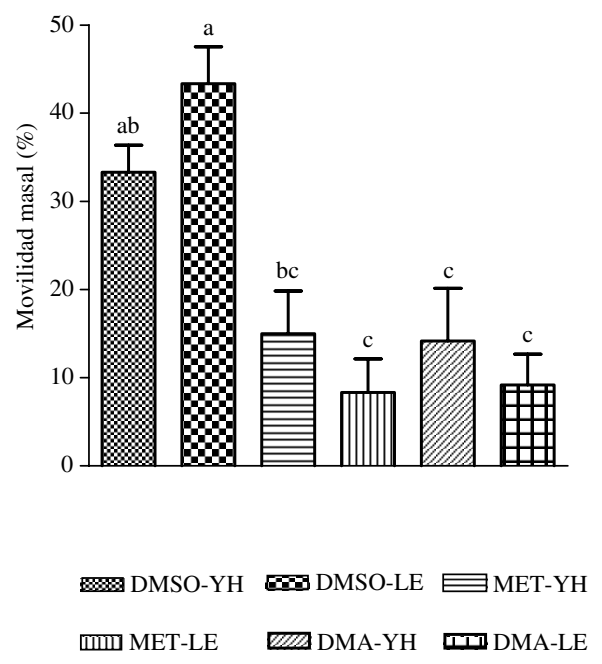

Figura 2. Variación de la movilidad espermática masal de semen de bagre rayado (Pseudoplatystoma metaense) durante las diferentes fases del proceso de crioconservación (a y c, fase 1, semen fresco $(n=11)$; fase 2, semen diluido (precongelación) $(n=3)$; fase 3, movilidad masal postdescongelación sometido a la $\mathrm{C} 1$ y C2 $(\mathbf{b}$ y d) $(\mathrm{n}=6)$. Valores corresponden a la media \pm error estándar de la media (EE). ${ }^{\mathrm{a}, \mathrm{b}, \mathrm{c}}$ Entre barras, valores con letras distintas son diferentes $(\mathrm{P}<0,05, \mathrm{n}=6)$.

Masal sperm motility of bagre rayado (Pseudoplatystoma metaense) during the cryopreservation. Assessment of motility in different phases (a and $\mathbf{c}$, phase 1, fresh semen ( $\mathrm{n}=11)$, phase 2, diluted semen (prefreezing) $(\mathrm{n}=3)$, Phase 3, masal post-thawing motility under the $\mathrm{C} 1$ and $\mathrm{C} 2$ (b and d) $(n=6)$. Values are the mean \pm standard error of the mean $(S E){ }^{a, b, c}$ Between bars, values with different letters are significantly different $(P<0.05, n=6)$.

YH-C1, DMSO-YH-C2, DMSO-LE-C1 y MET-YH-C1. Los tratamientos MET-YH-C1 y DMA-LE-C1 mostraron cabezas degeneradas (d). Por otro lado, todos los tratamientos mostraron la presencia de flagelos fracturados (j) excepto en los tratamientos DMSO-YH-C2, DMSOLE-C2 y DMA-YH-C2.

\section{ENSAYOS DE FERTILIDAD}

A pesar que las movilidades postdescongelación fueron menores a las determinadas con el SF y en algunos casos no se observó movilidad, tanto subjetivamente como en la evaluación con el CASA, se pudo evidenciar algún grado de capacidad fecundante en todos los tratamientos. Los porcentajes de fecundación menores se observaron para el semen sometido a congelación con la $\mathrm{C} 1$, en contraste con lo mostrado por la $\mathrm{C} 2$, donde la fertilidad fue superior al $60 \%$ (figura 5), con valores mayores para los tratamientos DMSO-LE-C2 $(79,6 \pm 2,1 \%)$ y DMA-LE-C2 $(75,6 \pm 7,3 \%)$, sin diferencias significativas $(\mathrm{P}>0,05)$ entre estos o cuando fueron comparados con el semen fresco $(88,4 \pm 4,0 \%)$.

\section{DISCUSIÓN}

En acuicultura, los beneficios de la crioconservación de gametos incluyen desde la posibilidad de selección y utilización de material genético de mayor calidad, hasta la reducción de los costos por mantenimiento de reproductores (Mongkonpunya y col 2000). Debido a las características de interés comercial y a la marcada estacionalidad reproductiva de Pseudoplatystoma metaense, se hace necesario 
a

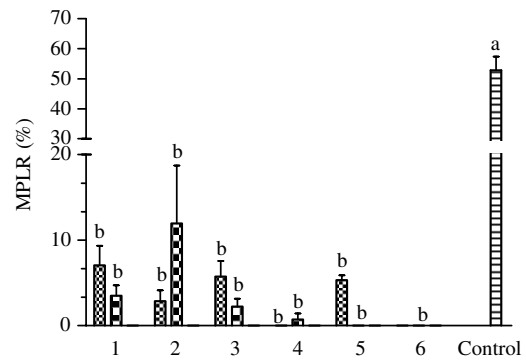

$\mathrm{C}$

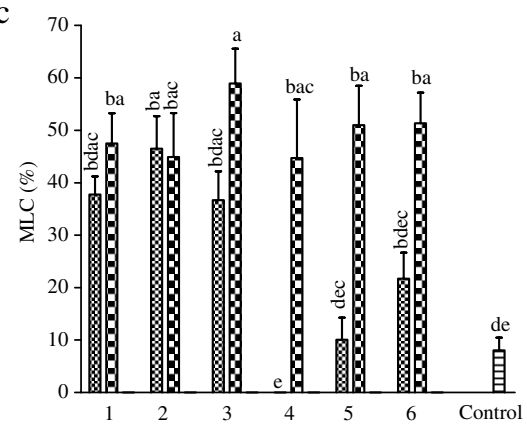

$\mathrm{b}$

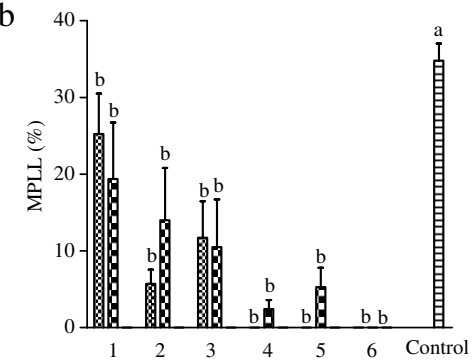

$\mathrm{d}$

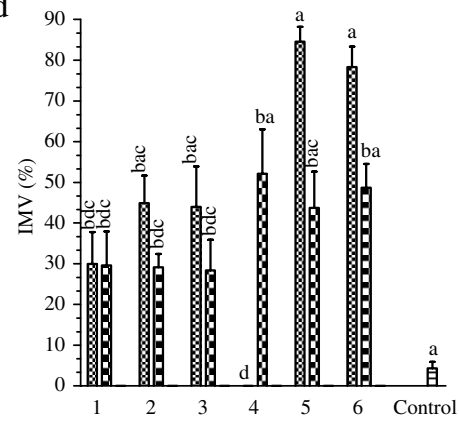

f

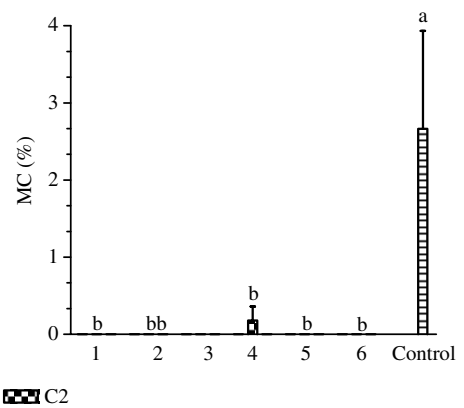

Figura 3. Movilidad individual postdescongelación determinada por CASA. (a) Movilidad progresiva lineal rápida (MPLR), (b) Movilidad progresiva lineal lenta (MPLL), (c) Movilidad local o nado círculo (MLC), (d) Inmóviles (IMV), (e) Movilidad total espermática individual (MTI), (f) Movilidad circular espermática (MC), para semen crioconservado de bagre rayado (Pseudoplatystoma metaense). Valores expresados como media \pm error estándar de la media (EE). ${ }^{\text {a,b,c,d,e }}$ Entre barras, valores con letras distintas son diferentes $(\mathrm{P}<0,05, \mathrm{n}=6)$.

Post-thawing individual motility determined by CASA. (a) Rapid linear progressive motility (MPLR), (b) Slow linear progressive motility (MPLL), (c) Local motility and swimming circle (MLC), (d) Immobile (IMV), (e) Motility total individual sperm (MTI) (f) Sperm motility circular (MC) for bagre rayado (Pseudoplatystoma metaense) cryopreserved semen. Values expressed as mean \pm standard error of the mean (SE). a,b,c,d,e Between bars, values with different letters are significantly different $(\mathrm{P}<0.05, \mathrm{n}=6)$.

optimizar la eficiencia de los protocolos de congelación de su material seminal; no obstante, en la actualidad los estudios en este campo son escasos. En el presente estudio el protocolo de crioconservación mostró influencia sobre la movilidad espermática postdescongelación: semen diluido con DMSO-YH y MET-YH y congelado lentamente (C1) mostró movilidad espermática mayor a 20\%, siendo muy baja cuando se utilizó MET y DMA como crioprotectores; sin embargo, la respuesta del semen sometido a crioconservación con una curva de congelación rápida $(\mathrm{C} 2)$ mostró movilidades espermáticas más altas, especialmente cuando se utilizó DMSO. Linhart y col (2005) reportaron para Silurus glanis porcentajes de movilidad inferiores a
12,1\% utilizando MET al 10\%. El uso de DMSO en la congelación seminal del bagre africano (Clarias gariepinus) mostró movilidades espermáticas cercanas a las observadas en este estudio (19\%), utilizando una curva una tasa de $4{ }^{\circ} \mathrm{C} \cdot \mathrm{min}^{-1}$ desde 3 hasta $-4^{\circ} \mathrm{C}$ y de $11{ }^{\circ} \mathrm{C} \cdot \mathrm{min}^{-1}$ desde -4 hasta $-80^{\circ} \mathrm{C}$ (Urbányi y col 1999), la cual corresponde a dos periodos de descenso: uno inicial lento, seguido por un periodo de descenso rápido, tal como la curva $\mathrm{C} 1$ utilizada en este ensayo. En las curvas de congelación lentas las células tienen más tiempo para deshidratarse antes de la congelación (Mazur 1970); sin embargo, esta exposición prolongada puede ocasionar la presencia de un medio residual hiperosmótico, que posiblemente influya sobre la 

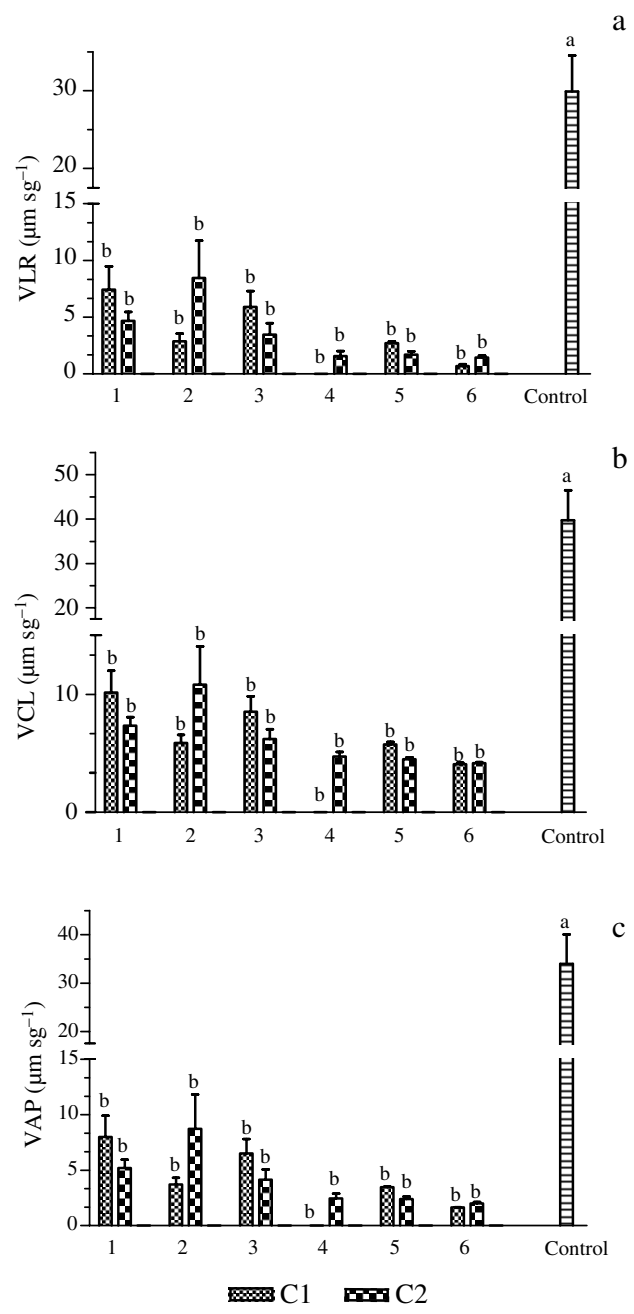

Figura 4. (a) Velocidad espermática en línea recta (VLR), (b) Velocidad espermática curvilínea (VCL) y (c) Velocidad promedio de desplazamiento (VAP) para semen crioconservado de bagre rayado (Pseudoplatystoma metaense), sometido a dos curvas de congelación (C1 y C2). 1(DMSO-YH); 2(DMSO-LE); 3(MET-YH); 4(MET-LE); 5(DMA-YH); 6(DMA-LE); control (semen fresco). Los valores son expresados como media \pm error estándar de la media (EE). ${ }^{\mathrm{a}, \mathrm{b}}$ Entre barras, valores con letras distintas son significativamente diferentes $(P<0,05, n=6)$.

(a) Sperm straight-line speed (VLR), (b) sperm curvilinear velocity (VCL) and (c) Average speed of travel (VAP) for bagre rayado (Pseudoplatystoma metaense) cryopreserved semen, subjected to two freezing curves (C1 and C2). 1 (DMSO-YH) 2 (DMSO-LE), 3 (MET-YH), 4 (TEM-LE), 5 (DMA-YH), 6 (DMA-LE), control (fresh semen).Values are expressed as mean \pm standard error of the mean $(\mathrm{SE})$. $^{\mathrm{a}, \mathrm{b}}$ Between bars, values with different letters are significantly different $(\mathrm{P}<0.05$, $\mathrm{n}=6)$.

viabilidad postdescongelación. Además, los cambios en la estructura y viabilidad de los espermatozoides durante el proceso de congelación son influenciados por la longitud del pico de calor latente, ocasionado por la liberación del calor de fusión durante el cambio de temperatura de -5 a $-45^{\circ} \mathrm{C}$ (Richardson y col 1999). En contraste a lo determinado en el presente estudio, en Pangasius larnaudii la

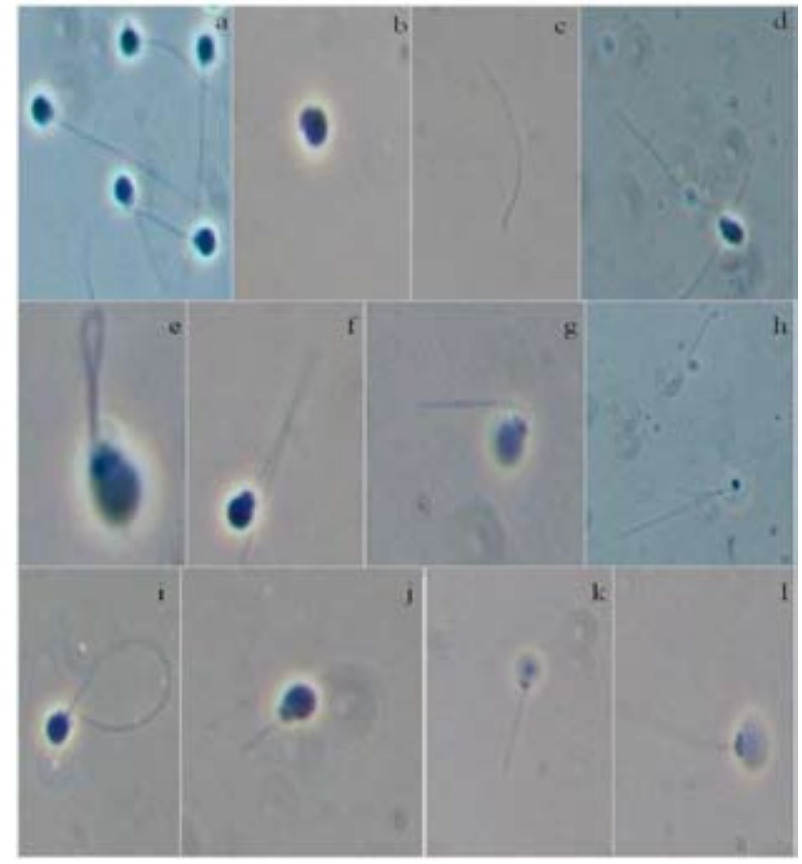

Figura 5. Alteraciones morfológicas encontradas en semen de bagre rayado (Pseudoplatystoma metaense) postdescongelación. a. espermatozoide normal; b. cabeza aislada; c. flagelo aislado; d. cabeza degenerada; e. flagelo doblado; f. flagelo fracturado; g. flagelo doblado; h. microcefalia y flagelo aislado; i. flagelo doblado; j. flagelo fracturado; $\mathbf{k}$ y $\mathbf{l}$. macrocefalia y flagelo fracturado. Observaciones en fresco (100X), realizadas en semen crioconservado diluido con una solución de formaldehido $35 \%$ (4\%)-citrato de sodio $\left(2,9 \%, \mathrm{Na}_{2} \mathrm{H}\left(\mathrm{C}_{3} \mathrm{H}_{5} \mathrm{O}(\mathrm{COO})_{3}\right)\right.$.

Morphological alterations found in bagre rayado (Pseudoplatystoma metaense) post-thawing semen. a. normal sperm; b. isolated head; c. isolated scourge; d. degenerate head; e. bent flagellum; f. flagellum broken; g. scourge bent; h. microcephaly and scourge isolated; i. scourge bent; $\mathbf{j}$. fractured scourge; $\mathbf{k}$ and $\mathbf{l}$. macrocephaly and fractured flagellum. Observations made in fresh (100X), diluted semen cryopreserved with a formaldehyde solution $35 \%$ (4\%)-Sodium citrate $\left(2,9 \%, \mathrm{Na}_{2} \mathrm{H}\left(\mathrm{C}_{3} \mathrm{H}_{5} \mathrm{O}\right.\right.$ $\left.(\mathrm{COO})_{3}\right)$

movilidad espermática masal fue superior cuando se utilizó DMSO al $10 \%$ y DMA al $10 \%(67,1 \pm 5,0$ y $58,6 \pm 5,0 \%$, respectivamente) con la inclusión de $\mathrm{NaCl}$ y una solución balanceada de calcio (Kwantong y Bart 2006) y utilizando una única tasa de descenso de temperatura: $10^{\circ} \mathrm{C} \cdot \mathrm{min}^{-1}$ de $25^{\circ} \mathrm{C}$ a $-80{ }^{\circ} \mathrm{C}$.

El semen de silúridos muestra un rango alto de sensibilidad a los crioprotectores utilizados en la crioconservación. En bagre de canal, el MET resultó ser el más eficiente para alcanzar altos porcentajes de movilidad postdescongelación (Tiersch y col 1994); sin embargo, en este estudio, el MET fue el segundo crioprotector en preservar una movilidad masal aceptable (figura 2). Viveiros (2005) atribuye la sensibilidad del semen a los procesos de crioconservación por los efectos tóxicos de los crioprotectores y por las posibles variaciones en la composición del plasma seminal existentes entre los bagres de cautiverio y los silvestres. 


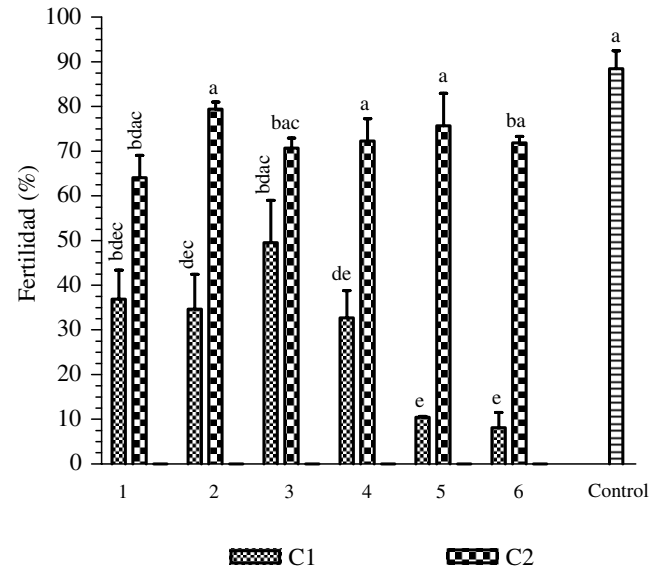

Figura 6. Porcentaje de fertilidad para semen crioconservado de bagre rayado (Pseudoplatystoma sp), sometido a dos curvas de congelación (C1 y C2). 1(DMSO-YH); 2(DMSO-LE); 3(MET-YH); 4(MET-LE); 5(DMA-YH); 6(DMA-LE); control (semen fresco). Los valores son expresados como media \pm error estándar (SE). ${ }^{\text {a,b,c,d,e }}$ Entre barras, valores con letras distintas son significativamente diferentes $(\mathrm{P}<0,05)(\mathrm{n}=6$ para $\mathrm{C} 1)$ y $(\mathrm{n}=4$ para C2).

Fertility percentage for bagre rayado (Pseudoplatystoma metaense) cryopreserved semen, subjected to two freezing curves $(\mathrm{C} 1$ and C2). 1 (DMSO-YH) 2 (DMSO-LE), 3 (MET-YH), 4 (TEM-LE), 5 (DMA-YH), 6 (DMA-LE), control (fresh semen). Values are expressed as mean \pm standard error (SE). ${ }^{\mathrm{a}, \mathrm{b}, \mathrm{c}, \mathrm{d}, \mathrm{e}}$ Between bars, values with different letters are significantly different $(\mathrm{P}<0.05)(\mathrm{n}=6$ for $\mathrm{C} 1)$ and $(\mathrm{n}=4$ for $\mathrm{C} 2)$.

La evaluación de la calidad seminal postdescongelación, específicamente la movilidad y velocidad individuales, han sido poco evaluadas en silúridos. Rurangwa y col (2001) reportaron en Clarias gariepinus una reducción significativa de la movilidad progresiva postdescongelación con valores entre 24 y 27\% usando DMSO y YH como diluyentes. Estos resultados son similares a los de este estudio, ya que el semen fresco mostró movilidad progresiva significativamente mayor que el semen crioconservado, aun utilizando DMSO y MET en combinación con YH y LE.

Por otra parte, para especies como Silurus glanis, Esox masquinongy, el uso de DMSO preservó la movilidad con valores de 20,9 y 39,6\%, respectivamente, en contraste a Oncorhynchus mykiss donde los valores fueron inferiores $(8,2 \%$ ) (Lahnsteiner y col 1996, Ciereszco y col 1999, Linhart y col 2005). En este estudio, espermatozoides congelados con MET y DMA mostraron movilidades inferiores cuando fueron comparados con los otros crioprotectores, seguido de porcentajes mayores al $80 \%$ de IMV para el caso de DMA (figura 3), cercano a lo reportado en Oncorhynchus mykiss $(89,6 \pm 8,4 \%)$. De igual forma, la VLR, VCL y VAP, fueron significativamente reducidas en semen crioconservado, en comparación al SF, con una reducción mayor a 50\%. En semen de Silurus glanis, la velocidad total del espermatozoide postdescongelación presentó rangos entre 37 a $85 \mu \mathrm{m} \cdot \mathrm{sg}^{-1}$ con altas velocidades $\left(105 \mu \mathrm{m} \cdot \mathrm{sg}^{-1}\right)$ al utilizar 7,5 a 10\% de MET (Linhart y col 2005); valores superiores a los observados para Pseudoplatystoma metaense, donde la velocidad para todos los tratamientos fue menor a $10 \mu \mathrm{m} \cdot \mathrm{sg}^{-1}$. De esta manera, los reportes confirman la obtención de resultados favorables con la incorporación de DMSO en la crioconservación de semen de peces teleósteos (Gwo 1999, Basavaraja y Hedge 2004), ampliamente recomendado para Pangasius gigas (Mongkonpunya y col 2000), Pangasius hypopthalmus (Kwantong y Bart 2003) y Clarias gariepinus (Horvath y Urbanyi 2000). Lo anterior se explica posiblemente por la alta tasa y rápida penetración del crioprotector en la célula espermática y por baja toxicidad de esta sustancia a bajas temperaturas (Lueng 1991). Sin embargo, Blottner (1989) reportó que las velocidades altas de congelación producen alteraciones fisiológicas, posiblemente por un evento similar a la precapacitación espermática observada en semen de mamíferos.

Los criterios utilizados para la evaluación de la calidad seminal en peces han sido basados en exámenes de movilidad, concentración espermática y porcentaje de espermatozoides vivos y muertos (Kavamoto y col 1985, Cruz-Casallas y col 2004), sin considerar los posibles defectos o alteraciones morfológicas en las células espermáticas. Estos estudios han alcanzado mayor importancia en la relación con la infertilidad de los machos y los eventos de inseminación artificial en mamíferos, especialmente en bovinos (Barth y Oko 1989), siendo poco estudiados en peces. Martins (2008), en semen crioconservado de Zungaro jahu, evidenció la presencia de alteraciones morfológicas, principalmente microcefalia en semen diluido con DMSO y YH, las cuales fueron también halladas en el presente estudio, predominando para todos los tratamientos la presencia de cabezas y flagelos aislados. Recientemente, Muchilisin y Siti (2009) observaron también anormalidades en semen congelado de Mystus nermurus, utilizando DMSO y MET (78,6 \pm 7,7 y 62,6 \pm 5,2\%, respectivamente) a nivel de la región de la cabeza y la presencia de ruptura y ausencia de flagelo, lo cual fue igualmente encontrado en el presente estudio.

Lahnsteiner y col (1992) reportaron que los cambios en la morfología espermática pueden producirse antes de la congelación o inmediatamente después del contacto con el diluyente, aumentando a medida que se prolonga el tiempo de exposición al crioprotector. En el presente trabajo, la combinación de MET-YH-C1 y DMA-LE-C1 condujo a la presencia de cabezas degeneradas. Según Martin (2008) este tipo de anormalidades morfológicas se caracteriza por cabeza de tamaño normal, pero con contornos irregulares y con disrupción de la cromatina. Lahnsteiner y col (1996) y Taddei y col (2001) asociaron esta alteración con una pérdida de funcionalidad de la mitocondria y de la cromatina nuclear, dado inicialmente por exposición de espermatozoides a soluciones crioprotectoras, acompañadas de inestabilidad en la osmorregulación de las células espermáticas. De esta manera, la evaluación de la magnitud de los daños morfológicos es muy importante para evitar la posible pérdida de la capacidad fecundante 
o la variabilidad genética durante el almacenamiento del esperma y entender las posibles alteraciones durante el desarrollo del embrión (Cabrita y col 2005).

En el presente trabajo todos los tratamientos mostraron fertilidad, con mayores porcentajes para el semen crioconservado con $\mathrm{C} 2$ y en especial para los tratamientos DMSO-LE-C2 y DMA-YH-C2, sin mostrar diferencias entre estos y el control. Estos resultados se asemejan a los observados para Clarias gariepinus (Viveiros y col 2000), en el cual se reportaron altas tasas de fecundación. No obstante, en otras especies como Pangasius larnaudii (Kwantong y Bart 2006) se observaron valores de fertilidad menores a $40 \%$ con MET al 10\% (7,9 $\pm 3,3 \%)$. Sin embargo, para otras especies de bagre como Pelteobagrus fulvidraco (Pan y col 2008) se reportaron movilidades postdescongelación de 4,0 $\pm 2,5 \%$ con una fertilidad de $90,4 \pm 3,6 \%$ usando MET 10\%. En el presente estudio, la movilidad no fue relacionada con las tasas de fertilidad (figuras 2 y 6). Por ejemplo, muestras con $0 \%$ de movilidad espermática (CASA) mostraron porcentajes de fertilidad alrededor del $10 \%$. Estos sucesos, probablemente se pueden atribuir a que un porcentaje de espermatozoides conserva intacta la estructura cromosómico contenida en el núcleo localizado en la cabeza y a las posibles interacciones entre espermatozoide-ovocito, lo cual debido a la existencia de un aparato micropilar en el ovocito de los peces permite que espermatozoides sin movilidad o presencia de vibración penetren al ooplasma, produciendo la fecundación del ovocito (Andrade y col 2001, Grassiotto y col 2001). Autores como Rana y col (1990) atribuyen este fenómeno a algunos factores de los ovocitos que pueden activar a aquellos espermatozoides inmóviles. De igual forma, Iwamatsu (2000) y recientemente Babin y col (2007) discutieron que sumado a factores ambientales, la presencia de agentes liberados por los ovocitos como pequeñas moléculas de polipéptidos sintetizados en el folículo de las células y acumuladas en el corion (factores iniciadores de la movilidad) pueden producir hiperactividad de la movilidad espermática en algunas especies de peces. Lo anterior podría sustentar parcialmente la presencia de fertilidad en ovocitos fecundados con espermatozoides inmóviles.

En conclusión, la combinación de 10\% DMSO, 5,5\% de glucosa más 5\% de LE o la mezcla de $12 \%$ MET, $5,5 \%$ de glucosa más $5 \%$ de LE, usando curvas rápidas de congelación (C2), pueden constituir un protocolo eficiente para la crioconservación seminal de Pseudoplatystoma metaense.

\section{RESUMEN}

El objetivo fue determinar el efecto de diferentes diluyentes y tasas de congelación sobre la calidad seminal de bagre rayado. Fueron utilizados machos $(1,5 \pm 0,2 \mathrm{~kg}$ y $56,6 \pm 2,9 \mathrm{~cm})$ y hembras sexualmente maduras $(2,8 \pm 0,7 \mathrm{~kg}$ y $71,3 \pm 6,1 \mathrm{~cm})$. La ovulación y espermiación fueron inducidas con Extracto de Hipófisis de Carpa. El semen fue diluido (1:6, semen: diluyente) con una solución a base de glucosa
$(5,5 \%)$ y yema de huevo (YH, $12 \%)$ o leche entera en polvo (LE, $5 \%)$. Como crioprotectores fueron utilizados dimetil sulfóxido (DMSO, $10 \%$ ), metanol (MET, 12\%) o dimetil acetamida (DMA, 10\%). El semen diluido fue empacado en pajillas de $0,5 \mathrm{~mL}$ y congelado bajo dos curvas de congelación $\left(\mathrm{C} 1=2,4{ }^{\circ} \mathrm{C} \cdot \mathrm{min}^{-1}\right.$ de $28 \mathrm{a}-4^{\circ} \mathrm{C}$, seguido por $10,0{ }^{\circ} \mathrm{C} \cdot \mathrm{min}^{-1} \mathrm{de}-4 \mathrm{a}-80{ }^{\circ} \mathrm{C}$ y C2 $=8,4{ }^{\circ} \mathrm{C} \cdot \mathrm{min}^{-1} \mathrm{de} 4 \mathrm{a}-80{ }^{\circ} \mathrm{C}$ ) y posteriormente sumergidos en nitrógeno líquido $\left(-196^{\circ} \mathrm{C}\right)$. Las pajillas fueron descongeladas en baño de agua a $35^{\circ} \mathrm{C}$ por 60 s e inmediatamente después evaluada la movilidad. La fertilidad fue evaluada a través de la seminación de 2 gramos de ovocitos con 400 ó $100 \mu \mathrm{L}$ de semen congelado o fresco, respectivamente, y determinada a las 6:00 h postfecundación. Los resultados mostraron mayores porcentajes de fertilidad con semen fresco, observándose ovocitos fecundados en todos los tratamientos, con mayor porcentaje para el semen sometido a la C2 (mayor al 60\%) y 10 ó $12 \%$ de DMSO o MET, respectivamente y $5,5 \%$ de glucosa más $5 \%$ de LE. Estos resultados permiten establecer un protocolo de congelación con resultados aceptables para la reproducción artificial de bagre rayado.

\section{AGRADECIMIENTOS}

Los autores agradecen al Ministerio de Agricultura y Desarrollo Rural (Contrato 015-1/06 MADR-CIAT-Universidad de los Llanos), así como al Instituto de Investigaciones de la Orinoquia Colombiana (IIOC) por el apoyo financiero y al Instituto de Acuicultura de la Universidad de los Llanos por el apoyo logístico.

\section{REFERENCIAS}

Andrade RF, N Bazzoli, E Rizzo, Y Sato. 2001. Continuous gametogenesis in the neotropical freshwater teleost, Bryconops affinis (Pisces: Characidae). Tiss Cell 33, 524-532.

Babin PJ, J Cerdá, E Lubzens, 2007. The fish oocyte: from basic studies to biotechnological applications. Springer, Dordrecht, The Netherlands.

Barth AD, RJ Oko. 1989. Abnormal morphology of bovine spermatozoa. Iowa State University, Ames, Iowa, USA.

Basavaraja N, SN Hegde. 2004. Cryopreservation of the endangered mahseer (Tor khudree) spermatozoa: I. Effect of extender composition, cryoprotectants, dilution ratio, and storage period on post-thaw viability. Cryobiology 49, 149-156.

Blottner S. 1989. Kapazitation und Akrosomenreaktion von Säugetierspermien und ihr Nachweis als befruchtungsbiologische Funktionsprüfung. Biol Zbl 108, 41-62.

Cabrita E, V Robles, L Rebordinos, L Sarasquete, MP Herráez. 2005. Evaluation of DNA damage in rainbow trout (Oncorhynchus mykiss) and gilthead sea bream (Sparus aurata) cryopreserved sperm. Cryobiology 50, 144-153.

Ciereszco A, K Dabrowski, F Lin, A Christ, GP Toth. 1999. Effects of extenders and time of storage before freezing on motility and fertilization of cryopreserved Muskellunge spermatozoa. Trans Am Fish Soc 128, 542-548.

Cruz-Casallas PE, SP Pardo-Carrasco, JA Arias-Castellanos, PE Lombo-Castellanos, DA Lombo-Rodriiguez, JE Pardo-Mariño. 2004. Cryopreservationof yamu' Brycon siebenthalae milt. J World Aquacult Soc 35, 529-535.

Darin-Bennet A, IG White. 1977. Influence of the cholesterol content of mammalian spermatozoa on susceptibility to cold-shock. Cryobiology $14,466-470$

De Leeuw FE, AM de Leeuw, JHG Den Dass, B Coenbranden, AJ Verkleij. 1993. Effects of various cryoprotective agents and membranestabilizing compounds on bull sperm membrane integrity after cooling and freezing. Cryobiology 30, 32-44.

Drokin S, H Stein, H Bartscherer. 1998. Effect of cryopreservation on the fine structure of spermatozoa of Rainbow trout (Oncorhynchus mykiss) and Brown trout (Salmo trutta F. Fario). Cryobiology 37, 263-270. 
Fabbrocini ASL, S Lavadera, Rispoli, G Sansone. 2000. Cryopreservation of Seabream (Sparus aurata) Spermatozoa. Cryobiology 40, 46-53.

Farrant J. 1970. Mechanisms of injury and protection in living cells and tissue at low temperatures, In: Smith AU (ed). Current Trends in Cryobiology. Plenum Press, New York, USA, Pp 139-152.

Grassiotto Q, JN Negräu, ED Carvalho, F Foresti. 2001. Ultraestructure of spermatogenic cells and spermatozoa in Hoplias malabaricus (Teleostei, Characiformes, Erythrinidae). J Fish Biol 59, 14941502.

Guerrero ACE. 2003. Treinamento alimentar de pintado Pseudoplatystoma coruscans (Agassiz, 1829): Sobrevivência, crescimento e aspectos econômicos. Dissertação (mestrado)-Centro de Aqüicultura, Universidade Estadual Paulista, Jaboticabal, Brasil.

Iwamatsu T. 2000. Fertilization in fishes. In: Tarin J, Cano A (eds). Fertilization in Protozoa and Metazoa Animals. Springer, Berlin, Germany, Pp 90-145.

Kavamoto ET, WF Silveira, MG Rigolino, AC Carvalho Filho. 1985. Avaliação macro e microscopic do semẽn da truta arco-íris, Salmo irideus Gibbons. Boletim do Instituto de Pesca 12, 73-81.

Kwantong S, AN Bart. 2006. Cryopreservation of black ear catfish, Pangasius larnaudii, (Bocourt) sperm. Aquacul Res 37, 955-957.

Lahnsteiner F, T Weismann, RA Patzner. 1992. Fine structure changes in spermatozoa of the grayling, Thymallus thymallus (Pisces: Teleostei), during routine cryopreservation. Aquaculture 103, 73-84.

Lahnsteiner F, B Berger, T Weismann, RA Patzner. 1996. Physiological and Biochemical Determination of Rainbow Trout, Oncorhynchus mykiss, semen quality for cryopreservation. J Appl Aquacul 5, 47-63.

Lahnsteiner F, B Berger, T Weismann, RA Patzner. 1996. Motility of spermatozoa of Alburnus alburnus (Cyprinidae) and its relationship to seminal plasma composition and sperm metabolism. Fish Physiolo and Biochem 15, 167-179.

Linhart O, M Rodina, M Flajshans, D Gela, M Kocour. 2005. Cryopreservation of European Catfish Silurus glanis sperm: Sperm motility, viability, and hatching success of embryos. Cryobiology $51,250-261$.

Lueng LKP. 1991. Principles of biological cryopreservation. In: Jamieson. Fish evolution: evidence from spermatozoa. Cambrigde Press, New York, USA, Pp 231-244.

Martins MD. 2008. Reprudução induzida de jaú (Zungaro jahu): análise das características seminais e ovocitárias. Dissertação em Zootecnia. Universidad Federal de Lavras, Lavras, Brasil.

Mazur P. 1970. Cryobiology: the freezing of biological systems. Science 168, 939-948.

Medina-Robles VM, YM Velasco-Satamaría, PE Cruz-Casallas. 2005. Aspectos generales de la crioconservación espermática en peces teleósteos. Rev Colomb Cienc Pecu 18, 34-48.

Medina-Robles VM, M Guarnizo-Pineda, JA Ramírez-Merlano, AM Otero-Paternina, T Mira, R Pacheco-Murillo, YM VelascoSantamaría, PE Cruz-Casallas. 2007. Caracterización y ensayos preliminares de crioconservación seminal de bagre rayado (Pseudoplatystoma fasciatum-Linnaeus, 1766). Memorias XIII Jornada de Acuicultura, Universidad de los Llanos, Villavicencio, Colombia, Pp 68-72.

Mojica Jl, C Castellanos, S Usma, R Álvarez. 2002. Libro rojo de peces dulceacuícolas de Colombia. La serie libros rojos de especies amenazadas de Colombia. Instituto de ciencias naturales. Universidad Nacional de Colombia, Ministerio del Medio Ambiente, Bogotá, Colombia.

Mongkonpunya K, T Pupipat, TR Tiersch. 2000. Cryopreservation of sperm of Asian catfishes, including the endangered Mekong giant catfish. In: Tiersch TR, Mazik PM (eds). Cryopreservation in Aquatic species. The World Aquaculture Society. Baton Rouge, Louisiana, USA, Pp 108-116.

Muchilisin ZA, AMN Siti. 2009. Influence of cryoprotectants on abnormality and motility of baung (Mystus nemurus) spermatozoa after long-term cryopreservation. Cryobiology 58, 166-169.

Ogier de Baulny B, Y Le Verne, D Kerboeuf, Maisse. 1997. Flow cytometric evaluation of mitchondrial activity and membrane integrity in fresh and cryopreserved Rainbow trout (Oncorhynchus mykiss) spermatozoa. Cryobiology 34, 141-149.

Ogier de Baulny B, C Labbé, G Maisse. 1999. Membrane integrity, mitochondrial activity, ATP content and motility of the European catfish (Silurus glanis) testicular spermatozoa after freezing with different cryoprotectans. Cryobiology 39, 177-184.

Pan J, S Ding, J Ge, W Yan, C Hao, J Chen, Y Huang. 2008. Development of cryopreservation for maintaining yellow catfish Pelteobagrus fulvidraco sperm. Aquaculture 279, 173-176.

Paul RL, KL Riley, JE Chandler, TR Tiersch. 2003. The use of dairy protocols for sperm cryopreservation of blue catfish Ictalurus furcatus. J World Aquacult Soc 34, 66-75.

Pinzón-Arciniegas SM, JE Mojica-Rodríguez, PE Cruz-Casallas. 2005. Ensayos preliminares sobre crioconservación de semen de bagre rayado (Pseudoplatystoma fasciatum Linnaeus, 1766). Orinoquia 9, 28-37.

Ramírez-Merlano JA, VM Medina-Robles, PE Cruz-Casallas. 2008. Crioconservación de semen de bagre rayado (Pseudoplatystoma fasciatum-Linnaeus, 1766) empleando un congelador programable: evaluación de la movilidad y fertilidad postdescongelación. En: memorias IV Congreso Colombiano de Acuicultura. Rev Colomb Cienc Pecu 21, 455-552.

Rana KJ, RM Muiruri, BJ McAndrew, A Gilmor. 1990. The influence of diluentes, equilibration time and pre-freezing storages time on the viability of cryopreservation Oreochromis niloticus (L.) spermatozoa. Aquacult and Fish Manag 21, 25-30.

Richardson GF, CE Wilson, LW Crim, XZ Yao. 1999. Cryopreservation of yellowtail flounder (Pleuronectes ferrugineus) semen in large straws. Aquaculture 174, 89-94.

Rurangwa E, FAM Volckaert, G Huyskens, DD Kime, F Ollevier. 2001. Quality control of refrigerated and cryopreserved semen using computer-assisted sperm analysis (Casa), viable staining and standardized fertilization in African catfish (Clarias gariepinus). Theriogenology 55, 751-769.

Taddei AR, F Barbato, L Abelli, S Canese, F Moretti, KJ Rana, AM Fausto, M Mazzini. 2001. Is cryopreservation a homogeneous process? Ultrastructure and motility of untrated, prefreezing, and postthawed spermatozoa of Diplodus puntazo (Cetti). Cryobiology $42,244-255$.

Urbányi B, A Horváth, Z Varga, L Horváth. 1999. Effect of extender on sperm cryopreservation of Arican catfish, Clarias gariepinus (Burchell). Aquacult Res 30, 145-151.

Villalobos-Sánchez MA, DM Osorio-Velandia. 2007. Ensayos preliminares de crioconservación de semen de Barbilla (Rhamdia sebae c.f.). Facultad de Ciencias Agropecuarias y Recursos Naturales, Universidad de los Llanos, Villavicencio, Colombia.

Viveiros ATM, N So, J Komen. 2000. Sperm cryopreservation of African catfish, Clarias gariepinus; cryoprotectans, freezing rates and sperm:egg dilution ratio. Theriogenology 54, 1395-1408.

Viveiros ATM. 2005. Semen cryopreservation in catfish species, with particular emphasis on the African catfish. Anim Breeding Abstr $73,1-9$. 